\title{
Levantamento Fitossociológico em Parcelas Permanentes na Floresta Nacional do Tapirapé-Aquiri, Pará, Brasil
}

\author{
Gleysla Gonçalves de Carvalho Fernandes ${ }^{1}$, Luana do Carmi Oliveira Ferreira ${ }^{1}$, \\ André Luís Macedo Vieira ${ }^{1} \&$ Álisson Rangel Albuquerque ${ }^{2}$
}

Recebido em 16/10/2020 - Aceito em 09/02/2021

${ }^{1}$ Instituto Chico Mendes de Conservação da Biodiversidade/ICMBio, Brasil <gleysla14gg@gmail.com, luana.itups@gmail.com,
andre.macedo@icmbio.gov.br>
${ }^{2}$ Universidade do Estado do Pará/UEPA, Brasil <alissonrangel@uepa.br>

RESUMO - O conhecimento da estrutura de uma floresta fornece subsídios para sua conservação e manejo. Nesse sentido, este trabalho objetiva realizar um levantamento fitossociológico em uma área com fitofisionomia de Floresta Ombrófila Aberta (FOA) na Floresta Nacional do Tapirapé-Aquiri, visando contribuir para planos de manejo com composições semelhantes, em seis parcelas permanentes $(0,25$ hectares cada). Em vista disso, foram identificadas e mensuradas todas as árvores com Diâmetro a Altura do Peito (DAP) $\geq 5 \mathrm{~cm}$ distribuídas em um intervalo de classes de $10 \mathrm{~cm}$, sendo calculados os índices de riqueza - Shannon ( $\left.\mathrm{H}^{\prime}\right)$ e Pielou (J), e fitossociológicos: frequência, dominância e densidade, relativas e absolutas, além do Valor de importância (VI) e cobertura (VC). A área apresentou uma diversidade de $H^{\prime}=3,89$ e uniformidade de $\mathrm{J}=0,58$, com $68 \%$ dos indivíduos distribuídos na primeira classe de diâmetro $(5-15 \mathrm{~cm})$, entre as mais representativas estão: Cenostigma tocantinum, Metrodorea flavida, Protium paniculatum e Attalea speciosa, por apresentarem os maiores valores de importância e cobertura, e a Bertholletia excelsa e C. catenaeformis por apresentarem os maiores valores de dominância. Essas espécies também apresentaram características interessantes para novas perspectivas de manejo, todavia, são necessários mais estudos ecológicos e socioeconômicos que fortaleçam esse conhecimento.

Palavras-chave: Conservação; ecologia; manejo sustentável; restauração florestal.

\section{Phytosociological and Floristic in Permanent Plots in the Tapirapé-Aquiri National Forest, Pará, Brazil}

ABSTRACT - Knowledge of the structure of a forest provides subsidies for its conservation and management. In this sense, this work aims to carry out a phytosociological survey in an area with Open Ombrophilous Forest (FOA) phytophysiognomy in the Tapirapé-Aquiri National Forest, aiming to contribute to management plans with similar compositions, in six permanent plots $(0,25$ hectare). Thus, all trees with diameter at breast height $(\mathrm{DBH}) \geq 5 \mathrm{~cm}$ distributed in a $10 \mathrm{~cm}$ class interval were identified and measured, and the richness indices - Shannon $\left(\mathrm{H}^{\prime}\right)$ and Pielou $(\mathrm{J})$ were calculated, and phytosociological: frequency, dominance and density, relative and absolute, in addition to the Value of importance (VI) and coverage (VC). The area presented a diversity of $H^{\prime}=3,89$ and uniformity of $J=0,58$, with $68 \%$ of the individuals distributed in the first diameter class $(5-15 \mathrm{~cm})$, among the most representative are: Cenostigma tocantinum, Metrodorea flavida, Protium paniculatum and Attalea speciosa, for presenting the highest values of importance and coverage, and Bertholletia excelsa and $C$. catenaeformis for presenting the highest values of dominance. These species also presented interesting characteristics for new management perspectives, however, more ecological and socioeconomic studies are needed to strengthen this knowledge.

Keywords: Conservation; ecology; sustainable management; forest restoration.

\section{Estudio Fitosociológico y Florístico en Parcelas Permanentes en el Bosque Nacional Tapirapé-Aquiri, Pará, Brasil}

RESUMEN - Comprender la estructura de un bosque, incluyendo áreas con poco conocimiento, apoya la expansión de la conservación y la gestión sostenida de esta comunidad. En este sentido, este trabajo tiene como objetivo estudiar la estructura florística y horizontal a través de parcelas permanentes que se instalaron en una región con fitonfisonía del bosque abierta (FOA) en el Bosque Nacional Tapirapé- 
Aquiri, con el objetivo de contribuir a planes de gestión con composición similar. El estudio se realizó en el municipio de Marabá, Estado de Pará, en seis parcelas permanentes (0,25 hectáreas cada una). Se midieron e identificaron individuos con diámetro a altura pecho (DAP) $\geq 5 \mathrm{~cm}$, siendo procesados en formulaciones de índices de riqueza (Shannon ( $\left.\mathrm{H}^{\prime}\right)$ y Pielou $(\mathrm{J})$ ), así como fitosociológicos (frecuencia, dominio y densidad, relativos y absolutos, y valor de importancia (VI)). Se muestrearon un total de 721 individuos pertenecientes a 127 especies, 45 familias y 98 géneros, con una diversidad de H'=3,88, uniformidad de $\mathrm{J}=0,55$. Las especies más representativas fueron Cenostigma tocantinum, Metrodorea flavida, Protium paniculatum, Attalea speciosa, y Bertholletia excelsa, por presentar los valores fitosociológicos más altos. Estas especies también ofrecen posibilidades de uso comercial no maderera condicionadas a sus semillas y frutos garantizados al área de perspectiva de la gestión sostenida. Entre ellos, $C$. tocantinum, $M$. flavida, y P.paniculatum, debido a la rápida dispersión y crecimiento, posiblemente se pueden utilizar en la recuperación de áreas con composición similar, sin embargo, es necesario conocer mejor sus características forestales.

Palabras clave: Conservación; ecología; Manejo sostenible; restauración forestal.

\section{Introdução}

A Amazônia legal ocupa, aproximadamente $6.000 .000 \mathrm{~km}^{2}$ de território, caracterizando o Brasil como um dos maiores repositórios da biodiversidade mundial (Oliveira \& Amaral, 2004; Fernandes et al., 2020). Porém, a intensidade de perturbações ambientais ocorrentes na região amazônica tem contribuído para a exaustão dessa diversidade, em vista que a maioria do ecossistema tem sido explorado de forma não sustentável, desencadeando a perda da cobertura florestal e da comunidade de espécies (Souza-Filho et al., 2016).

A dinâmica natural da Amazônia coincide para que espécies respondam aos gradientes ambientais e criem uma diversidade de indivíduos mantidos localmente (Eiserhardt et al., 2011). Esses indivíduos são alvos de constante pressão antrópica pela substituição da vegetação nativa por atividades como a pecuária e a soja. Parte dessas atividades não são realizadas de forma sustentável, sendo o principal motivo para o Estado do Pará se caracterizar como um dos mais desmatados nos últimos sete anos (Carneiro et al., 2020). Nesse sentido, a ciência da conservação tem se desenvolvido com o intuito de tentar resolver esses problemas que ameaçam a biodiversidade, criando respostas eficazes para o manejo sustentável (Rodríguez-Estrella \& Moreno, 2006).

Na região da Serra dos Carajás, há um conjunto de unidades de conservação (UCs) que concilia a conservação e o uso sustentável de seus bens e serviços naturais. Uma delas é a Floresta Nacional do Tapirapé-Aquiri (FLONATA), que compreende 196.351,42 hectares de território dominado por duas tipologias, Floresta Ombrófila Aberta (FOA) $(86 \%)$ e resquícios de Floresta Ombrófila Densa (FOD) (15\%). Ambas são similares, mas se diferem na composição florística, em que a FOA apresenta menor densidade de grandes árvores e maior ocorrência de palmeiras e lianas (Gama et al., 2007).

Essas informações sobre a composição florística permitem conhecer e estudar aspectos ecológicos que são importantes para a criação de subsídios para valoração e conservação da floresta em pé. Por isso, a utilização de parcelas nesta fitofisionomia é largamente utilizada (Colpini et al., 2009; Xavier et al., 2011; Fernandes et al., 2020), visando a obtenção de informações florísticas e ecológicas, uma vez que elas permitem acompanhar e avaliar as mudanças de composição e sintetizar informações por diferentes padrões de riqueza (Joly et al., 2012).

$\mathrm{O}$ uso de parcelas permanentes possibilita o avanço da conservação com base na própria determinação da estrutura florestal (Malhi et al., 2002). Logo, derivado do pouco conhecimento que há sobre a estrutura e a dinâmica florestal da FOA da FLONATA, sua instalação e sucessivas medições tornam-se imprescindíveis na conservação e manejo racional de sua vegetação nativa.

Essa compreensão da composição e da diversidade de espécies é fundamental para entender o meio ambiente envolto, identificar novas perspectivas de manejo sustentável, além de suas próprias características ecológicas. Diante dos pressupostos, o presente trabalho objetiva realizar um levantamento fitossociológico de uma área de FOA na FLONATA, visando contribuir para os planos de manejo com composições semelhantes. 


\section{Material e Métodos}

\section{Área de estudo}

Este estudo foi desenvolvido na FLONATA, especificamente em uma área denominada de rio Cinzento, localizada no município de Marabá, estado do Pará (Fig. 1). Essa área apresenta uma topografia que varia de plana a plana associada à baixa declividade, sendo constituída por uma vegetação de FOA composta por um dossel descontínuo com grande presença de palmeiras.

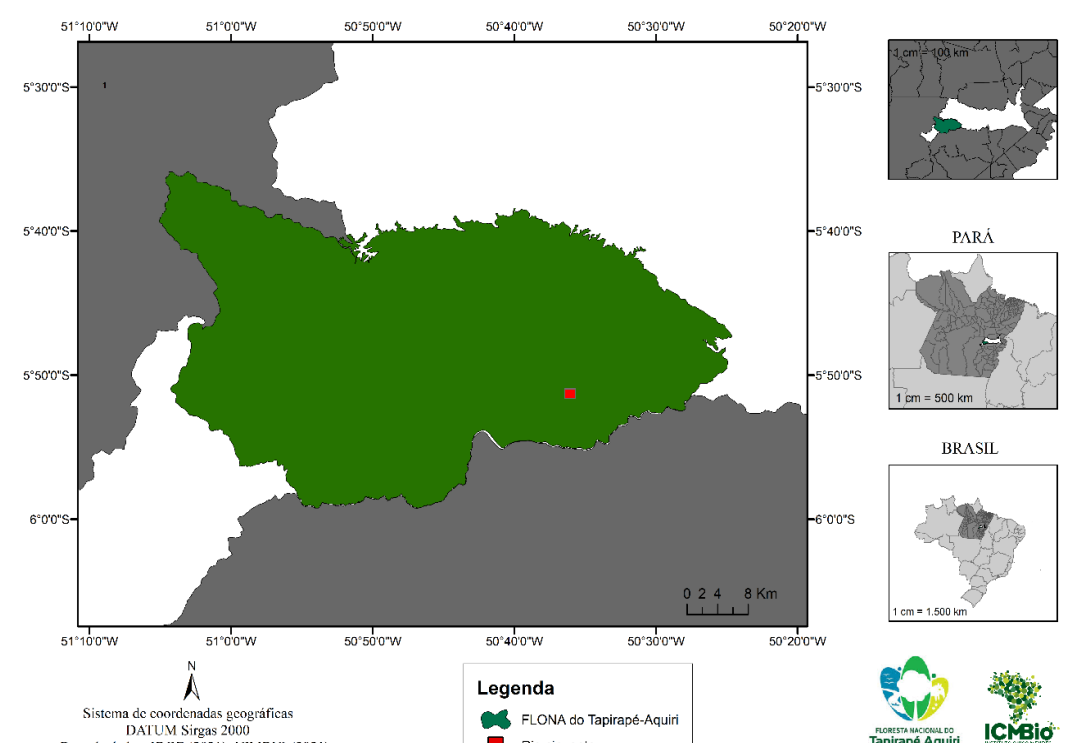

Figura 1 - Localização da Floresta Nacional do Tapirapé-Aquiri e das parcelas permanentes, no município de Marabá, estado do Pará, Brasil, 2021.

Entre as espécies mais importantes dessa fitofisionomia, destaca-se a Attalea speciosa Mart., seguida pelos indivíduos de Cenostigma tocantinum Ducke, Attalea maripa (Aubl.) Mart., e com grandes diâmetros, Bertholetia excelsa Bonpl (Brasil, 2006; Salomão et al., 2007).

Quanto ao clima da região de estudo, segundo a classificação climática de Koppen, é do tipo "awi", tropical chuvoso com seca de inverno e temperatura mensal sempre acima de $18^{\circ} \mathrm{C}$ com precipitação média anual de 1930,01 milímetros (Brasil, 2006), tendo como tipologia pedológica predominante o latossolo vermelho-amarelo (Fernandes et al., 2020).

\section{Coleta de dados}

Idealizado pela FLONATA em 2018, o projeto inventário surge com o intuito de inventariar uma região ainda pouco conhecida, com a instalação e medição de parcelas permanentes, visando a criação de um arcabouço florístico que futuramente será disponibilizado para a sociedade. Desse modo, para o inventário, foram instaladas de forma sistemática seis parcelas permanentes em 2018, medindo 50m x 50m (0,25ha cada).
As parcelas estão distantes entre si por $350 \mathrm{~m}$ e com diferentes distancias da picada principal, sendo denominadas de acordo com a instalação (P1, P2, e assim sucessivamente). Essas unidades foram demarcadas por piquetes (canos de PVC) e subdivididas em 25 subparcelas de $10 \mathrm{~m} \times 1 \mathrm{~m}$ (cada) para facilitação da coleta de dados (Fig. 2). Nas subparcelas, todos os indivíduos que apresentaram o diâmetro a altura do peito (DAP) maior ou igual a 5 centímetros foram mensurados. Essas mensurações consistiram de observações da vegetação ao longo das parcelas, quanto ao seu diâmetro e grau de penetração da luz, prezando obter informações da comunidade de estudo. As árvores mortas não foram identificadas, nem medidas.

Os indivíduos foram identificados por parabotânicos ligados ao Herbário da Floresta Nacional de Carajás (FLONAC), localizado no Parque Zoobotânico da FLONAC, no sudeste do Pará. Árvores que não puderam ser identificadas em campo, tiveram seu material reprodutivo coletado e levado ao Herbário de Carajás para identificação posterior. 


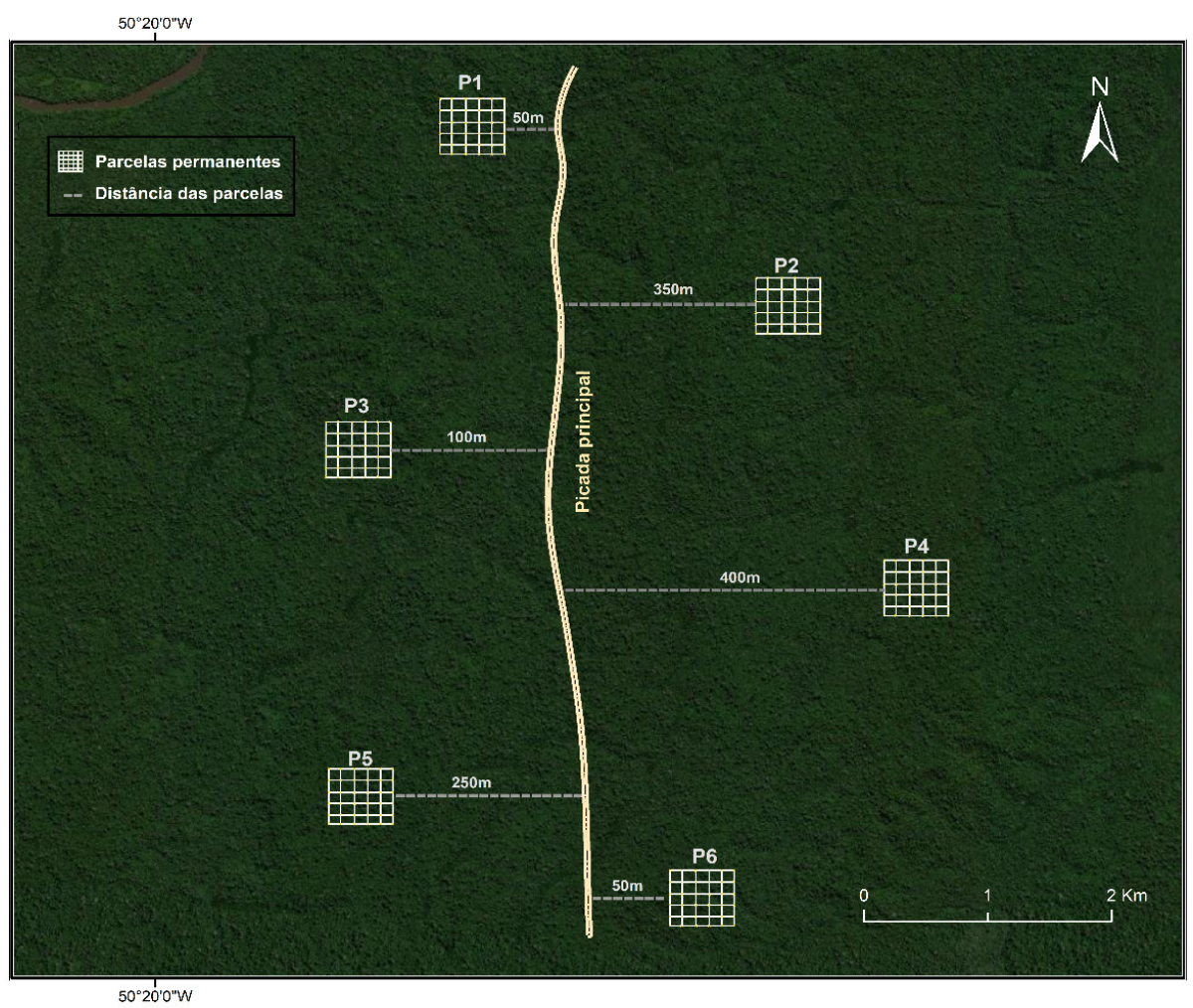

Figura 2 - Destaque para as parcelas permanentes instaladas na área rio Cinzento, Floresta Nacional do Tapirapé-Aquiri.

\section{Análise dos dados}

A comunidade florística foi analisada conforme as características de gêneros, espécies $e$ familias. Espécies e familias foram consideradas importantes segundo os resultados da estrutura horizontal e localmente raras com base em seu tamanho populacional, seguindo os conceitos de "singletons" (espécies representadas por apenas um indivíduo) e "dobletons" (espécies representadas por dois indivíduos) (Gaston, 1994; Ferreira et al., 2015).

A análise da taxa de crescimento foi realizada mediante a distribuição diamétrica das árvores, distribuídas em 7 classes com intervalo de classes de $10 \mathrm{~cm}(5-15 \mathrm{~cm} ; 15-25 \mathrm{~cm} ; 25-35 \mathrm{~cm} ; 35-45 \mathrm{~cm}$; $55-65 \mathrm{~cm} ; 65-75 \mathrm{~cm}$; $\geq 500 \mathrm{~cm})$.

Para a avaliação da diversidade de espécies, foi utilizado o índice de riqueza de ShannonWiener (Magurran, 1988), obtido pela Equação 1.

$$
H^{\prime}=-\sum\left(p_{i}\right) \ln p_{i} \quad(1)
$$

Em que: $\mathrm{pi}=(\mathrm{ni} / \mathrm{N})$ é a probabilidade de que um indivíduo amostrado aleatoriamente pertença a espécie i; ni = no total de indivíduos da espécie i; e $\mathrm{N}=$ no total de indivíduos amostrados na área; ln = logaritmo neperiano.
E para a avaliação da equabilidade (uniformidade) foi utilizado o cálculo de Pielou (Pielou, 1966) pela Equação 2.

$$
J=H^{\prime} / H \max
$$

Em que: H' é o índice de diversidade de Shannon-Wiener e Hmax' =ln (S), sendo S o número total de espécies amostradas.

\section{Avaliações estruturais}

Os parâmetros da fitossociologia horizontal seguiram as recomendações de Mueller-Dombois \& Ellenberg (1974) para estimativa da densidade absoluta (DAi) e relativa (DRi), frequência absoluta (FAi) e relativa (FRi), dominância absoluta (DoAi) e relativa (DoRi), valor de importância (VI) e o valor de cobertura (VC). A estimativa da importância ecológica das famílias foi feita mediante ao Valor de importância familiar (VIF) ( $\mathrm{n}^{\circ}$ de espécies da família $/ \mathrm{n}^{\circ}$ total de espécies). Os cálculos foram realizados pelo Sistema de Monitoramento de Florestas Tropicais (MFT) utilizando as fórmulas expressas no Quadro 1. 
Quadro 1 - Descrições fitossociológicas para o estudo da estrutura horizontal da comunidade estudada.

\begin{tabular}{|c|c|}
\hline Fórmulas & Condições \\
\hline $\begin{array}{c}\text { DAi }=\frac{N_{i}}{h a} \\
\text { DRi }=\frac{\text { DAi }}{N} \times 100\end{array}$ & $\begin{array}{l}\text { Em que: } \\
\mathrm{DAi}=\text { densidade absoluta da i-ésima espécie; } \mathrm{DRi}=\text { densi- } \\
\text { dade relativa da i-ésima espécie; } \\
\mathrm{Ni}=\text { número de indivíduos da i-ésima espécie; } \mathrm{N} \text { = número } \\
\text { total de indivíduos }\end{array}$ \\
\hline $\begin{array}{c}\text { DoAi }=\frac{A B_{i}}{h a} \\
\text { DoRi }=\frac{\text { DoA }}{G} \times 100\end{array}$ & $\begin{array}{l}\text { Em que: } \\
\text { DoAi= dominância absoluta da i-ésima espécie; } \mathrm{DoRi}= \\
\text { dominância relativa da i-ésima espécie; } \mathrm{ABi}=\text { área basal } \\
\text { (somatório das áreas seccionais) da i-ésima espécie; } \mathrm{G}= \\
\text { área basal total }\end{array}$ \\
\hline $\begin{aligned} \mathrm{FA} & =\frac{\mathrm{np}}{\mathrm{NP}} \times 100 \\
\mathrm{FR} & =\frac{\mathrm{FA}}{\sum \mathrm{FA}} \times 100\end{aligned}$ & $\begin{array}{l}\text { Em que: } \\
\text { FAi = frequência absoluta da i-ésima espécie; FRi = frequên- } \\
\text { cia relativa da i-ésima espécie; } \\
\mathrm{np}=\text { número de unidades amostrais com ocorrência das } \\
\text { espécies; } \mathrm{NP}=\text { número total de unidades amostrais; } \sum \mathrm{FA}= \\
\text { somatório da frequência absoluta }\end{array}$ \\
\hline $\begin{array}{l}\mathrm{VC}=\mathrm{DR}+\mathrm{DoR} \\
\mathrm{VC}(\%)=\frac{\mathrm{VC}}{2}\end{array}$ & $\begin{array}{l}\text { Em que: } \\
\text { VC = valor de cobertura; } \mathrm{VC}(\%)=\text { valor de cobertura em } \\
\text { porcentagem; } \mathrm{DR}=\text { densidade relativa; DoA = dominância } \\
\text { relativa }\end{array}$ \\
\hline $\begin{array}{c}V I=D R i+D o R i+F R i \\
V I(\%)=\frac{V I}{3}\end{array}$ & $\begin{array}{l}\text { Em que: } \\
\mathrm{VI}=\text { valor de importância; } \mathrm{VI}(\%)=\text { valor de importância } \\
\text { em porcentagem; DRi = densidade relativa da i-ésima espé- } \\
\text { cie; DoRi = dominância relativa da i-ésima espécie; FRi = } \\
\text { frequência relativa da i-ésima espécie. }\end{array}$ \\
\hline
\end{tabular}

Fonte: Adaptado de Mueller-Dombois \& Ellenberg (1974).

\section{Resultados e Discussão}

\section{Composição florística}

Foram amostrados 784 indivíduos pertencentes a 133 espécies, 89 gêneros e 45 famílias, com uma média de 130 a 140 indivíduos por parcela. As famílias com maior número de espécies foram a Fabaceae (23), seguida pela Moraceae, Chrysobalanaceae, Burseraceae (7), Rutaceae (6), Annonaceae e Malvaceae (5), que responderam a quase $50 \%$ das espécies identificadas, sugerindo que a diversidade vegetal tem se concentrado em poucas famílias botânicas (Silva et al., 2015; Moura et al., 2017).

Treze famílias (Ochnaceae, Papilonaceae, Polygonaceae, Rhamnaceae, Rubiaceae, Ulmaceae, Urticaceae, Araliaceae, Clusiaceae, Elaecarpaceae, Euphorbiaceae, Humiraceae, Combretaceae, Myristicacceae) foram representadas por apenas uma espécie, 10,23\% do total de espécies e $28,88 \%$ do total de famílias. Desse conjunto, as que apresentaram raridade foram as famílias Araliaceae, Ochnaceae,
Combretaceae, Polygonaceae, Humiraceae, e Myristicaceae, com apenas um indivíduo. Considerando que essas não são famílias monoespecíficas (de apenas uma espécie), essa baixa densidade pode estar relacionada a amostragem, possivelmente, necessitavam de uma amostragem mais significativa para localização dos seus indivíduos.

Quanto à raridade das espécies, as parcelas amostradas tiveram $44,24 \%$ dos indivíduos considerados localmente raros, como a Buchenavia congesta Ducke, Scheffera morototoni (Aubl.), e Ouratea castaneifolia (DC.) Engl. Destacam-se ainda nesse padrão espécies vulneráveis, como a Apuleia leiocarpa (Vogel) J.F. Macbr, Hymenolobium excelsum Ducke, Anaxagorea brevipes Benth., Virola surinamensis (Rol. Ex Rottb.) Warb., e Talisia subalbens (Mart.) Radlk (Centro Nacional de Conservação da Flora (CNCFlora).

A raridade dessas espécies pode ser vista como um mecanismo de defesa contra a ação de predadores; tendo distribuição mais esparsa, há 
mais chances de obterem êxito na perpetuação de seus indivíduos (Ferreira et al. 2015). Por outro lado, essa condição implica na formação de um grupo mais vulnerável à extinção, tornando os indivíduos mais sensíveis e especializados em um conjunto restrito de fatores ambientais ou limitados em sua dispersão.

Analisando a existência desses indivíduos raros, é interessante apontar que todos os indivíduos supracitados apresentam potencial madeireiro, porém, devido à sua sensibilidade, são necessárias técnicas adequadas de manejo, a fim de não afetar o seu desenvolvimento populacional. Além disso, pela área de estudo ser uma UC, esse cuidado precisa ser ainda maior, uma vez que, segundo Souza \& Sccoti (2020), é um local que abriga não só espécies ameaçadas, mas também grande parte da biodiversidade local.

Quanto à diversidade de Shannon, o valor para a comunidade de estudo foi de $\mathrm{H}=3,89$ nats.indivíduo-1, considerado um valor médio, segundo Finol (1975), entretanto, baixo, uma vez que esse resultado na Amazônia tende a variar de 3,88 a 5,51 nats.indivíduo-1 (Oliveira et al., 2008; Brandão et al., 2020). Entre os fatores que podem ter influenciado para obtenção desse resultado pouco expressivo, estão os critérios de inclusão de indivíduos, a área total amostrada ou a própria abundância de espécies (Salles \& Schiavini, 2007).

Em relação à equabilidade de Pielou (J), o valor apresentado foi de $\mathrm{J}=0,58$, ou seja,
$58 \%$ da diversidade máxima, o que sugere uma uniformidade média nas proporções indivíduos/ espécies, indicando que ainda existe uma certa dominância de uma ou poucas espécies dentro da comunidade vegetal (Oliveira \& Amaral, 2004).

\section{Distribuição diamétrica}

Quanto ao estabelecimento diamétrico, 68\% dos indivíduos pertenceram a primeira classe de diâmetro (5 a $15 \mathrm{~cm})$, enquanto a segunda classe (15 a $20 \mathrm{~cm}$ ) apresentou $25 \%$ dos indivíduos (Fig. 3). Tendo em vista essa distribuição, menos de $0,63 \%$ da comunidade estudada apresentou um diâmetro superior a $500 \mathrm{~cm}$, os únicos indivíduos dessa classe foi a Bertholletia excelsa Bonpl. e a Cedrelinda catenaeformis L. O comportamento da curva foi em formato decrescente conhecido como "J-invertido", típico de muitas florestas nativas da Amazônia que decrescem ao maior diâmetro encontrado (Oliveira et al., 2008; Dionisio, 2020).

Por outro lado, a existência de diâmetros superiores a $500 \mathrm{~cm}$ corrobora o entendimento que a comunidade estudada se encontra em um alto estado de conservação. Isso é reforçado pela existência de duas espécies com alta vulnerabilidade (B. excelsa e $C$. catenaeformis) e que, pressupondo, tendem a manter os grandes diâmetros pelo favorecimento de ambientes pouco antropizados.

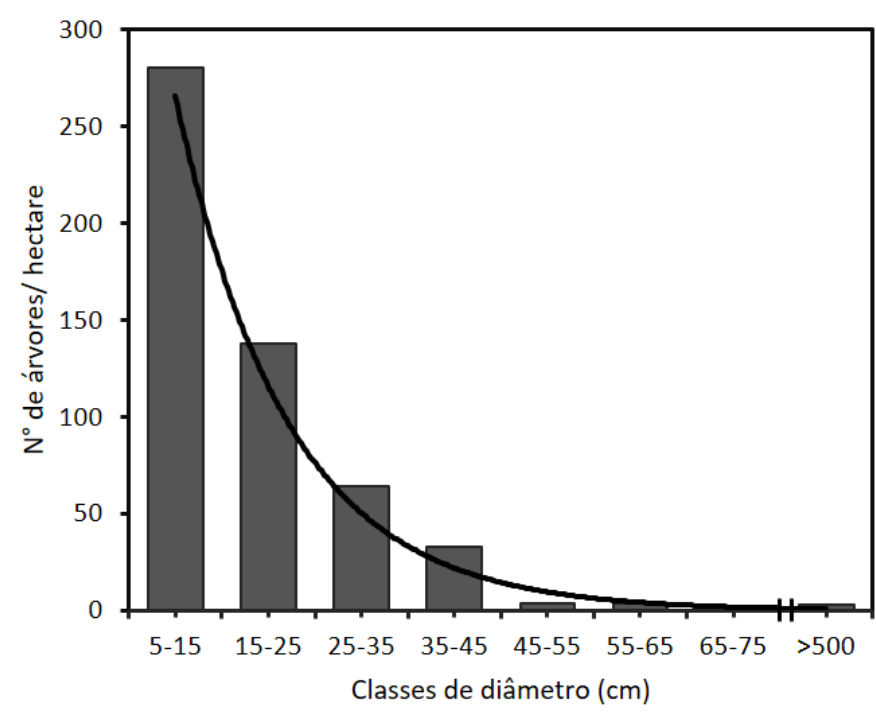

Figura 3 - Distribuição diamétrica de 784 indivíduos amostrados com o DAP $\geq 5 \mathrm{~cm}$ na área rio Cinzento. 


\section{Parâmetros fitossociológicos e perspectivas de uso sustentável}

Analisando as espécies mais abundantes, destacam-se a Cenostigma tocantinum Ducke (15,68\% dos indivíduos levantados), Metrodorea flavida K. Krause (10,14\%), Protium paniculatum (Engl.) (8,03\%) e Attalea speciosa Mart (6,00\%), totalizando $40 \%$ dos indivíduos amostrados. Essas espécies também foram as mais frequentes na área de estudo (Fig. 4A), confirmando estudos realizados em diferentes áreas da Amazônia que destacam a sua representatividade (Gama et al., 2007; Xavier et al., 2011, Fernandes et al., 2020; Lima et al., 2021).

Analisando o VIF, as famílias mais importantes foram: Fabaceae (25\%), Burseraceae (13\%), Chrysobalanaceae (10\%) e Malvaceae (10\%), com mais de $50 \%$ do valor de importância familiar, indicando sua importância ecológica para o equilibro florístico. Ao levar em conta o valor de indivíduos e o índice de importância, as duas famílias mais bem distribuídas foram Fabaceae e Burseraceae, ambas são características para florestas de terra firme (Oliveira \& Amaral 2005; Salomão, 2007; Burgh Daly, 2012), onde aparecem entre as mais diversificadas.

No que diz respeito à densidade de indivíduos, o valor estimado foi de 513,3 inds.ha-1, com uma área basal de $625,3 \mathrm{~m} 2$ ha-1. As espécies que apresentaram a maior densidade (DAi) foram: $C$. tocantinum (82,00 árvores/ha); M. flavida (42,00 árvores/ha); e P. paniculatum (31,33 árvores/ha). Em contrapartida, as espécies mais dominantes (DoAi) foram Bertholletia excelsa Bonpl., com 221,18 m2.ha-1 (4 indivíduos), a maior árvore identificada, Cedrelinda catenaeformis L., com 85, $78 \mathrm{~m} 2$.ha-1 (2 indivíduos), A. speciosa com 50, $90 \mathrm{~m} 2$.ha-1 (40 indivíduos) e C. tocantinum com 13,8 m2.ha-1 (123 indivíduos) (Fig. 4B).

Observa-se que a $B$. excelsa e a $C$. catenaeformis não são indivíduos representativos, apresentando baixa densidade relativa $(0,38 \%$, respectivamente). Um aspecto interessante é que o grande porte dessas espécies foi o que contribuiu para o destaque das mesmas no alto valor de área basal, e essa pouca representatividade decorre de fatores já citados como a amostragem, a limitação de dispersão, ou a própria característica da espécie, sendo um padrão demonstrado por alguns estudos realizados em florestas primarias (Hubbel \& Foster, 1985; Miller \& Kauffman, 1998; Nascimento et al., 2004; Lau et al., 2020).

Nesse cenário, é importante destacar que a B. excelsa é um indivíduo com uma distribuição alógama e com sistema de autoincompatibilidade (Cavalcante, 2008). Ainda assim, essa espécie é muito utilizada na economia de muitas comunidades tradicionais, pois oferece amêndoas que são apreciáveis para produção de polpas e consumo in natura (Schwartz, 2007). Diante dos pressupostos, essa utilização deve ser feita pensando na densidade populacional baseada em práticas silviculturais diferenciadas para o manejo e conservação desta (Nascimento et al., 2004).
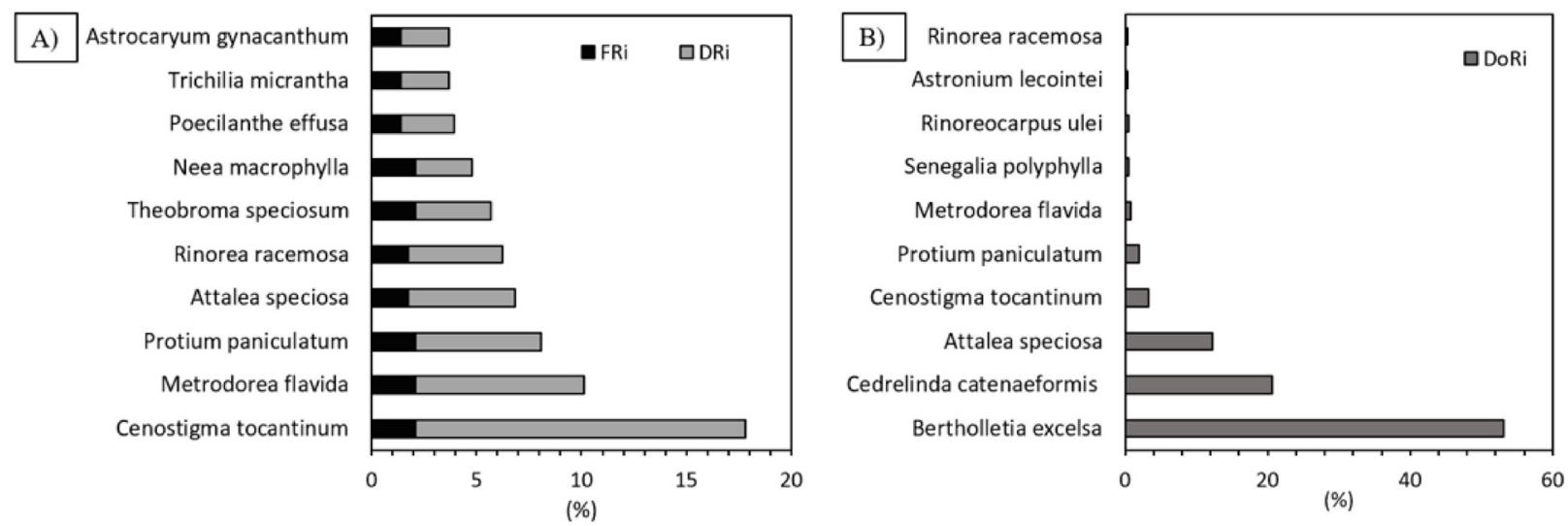

Figura 4 - Principais espécies para os parâmetros fitossociológicos levantados na área rio Cinzento, Floresta Nacional do Tapirapé-Aquiri, Pará, Brasil. A) espécies com maior densidade e frequência; B) espécies com maior dominância. 
Quanto à participação de espécies na comunidade, as palmeiras são um grupo presente tanto na dominância, quanto na densidade de seus indivíduos. Dos 784 indivíduos levantados, elas contribuíram com $17 \%$, sendo cerca de $5 \%$ desses indivíduos importantes ecologicamente. Comparando a levantamentos florísticos, esse padrãoé esperado, uma vez queéalgo característico para a tipologia de estudo ter uma concentração de árvores com menor DAP (Oliveira et al., 2008; Fernandes et al., 2020; Dionisio et al., 2020), além da própria análise de diâmetros menores favorecer o levantamento dessas espécies.

Essa abundância de palmeiras em uma área possibilita o seu enriquecimento em relação aos aspectos alimentares, medicinais e socioeconômicos
(Rocha \& Silva, 2005; Negrelle, 2015). Miranda et al. (2001) consideram as palmeiras na Amazônia como um dos recursos vegetais mais úteis para o homem e de maior interesse para o manejo por terem tantas possibilidades de uso.

Observando a amostragem de espécies quanto a sua importância ecológica, destacam-se com maior VI a C. tocantinum (8,89\%), M. flavida $(5,06)$, P. paniculatum $(4,04)$, A. Speciosa $(3,42 \%)$, Rinorea racemosa (Mart.) Kuntze (3,10\%), Theobroma speciosum Willd. ex Spreng. (2,83\%), que juntas contribuíram com $27 \%$ do VI analisado. Essas mesmas espécies se sobressaíram nos maiores valores de cobertura (VC) (Fig. 5), representando $38 \%$ do total de VC amostrado.

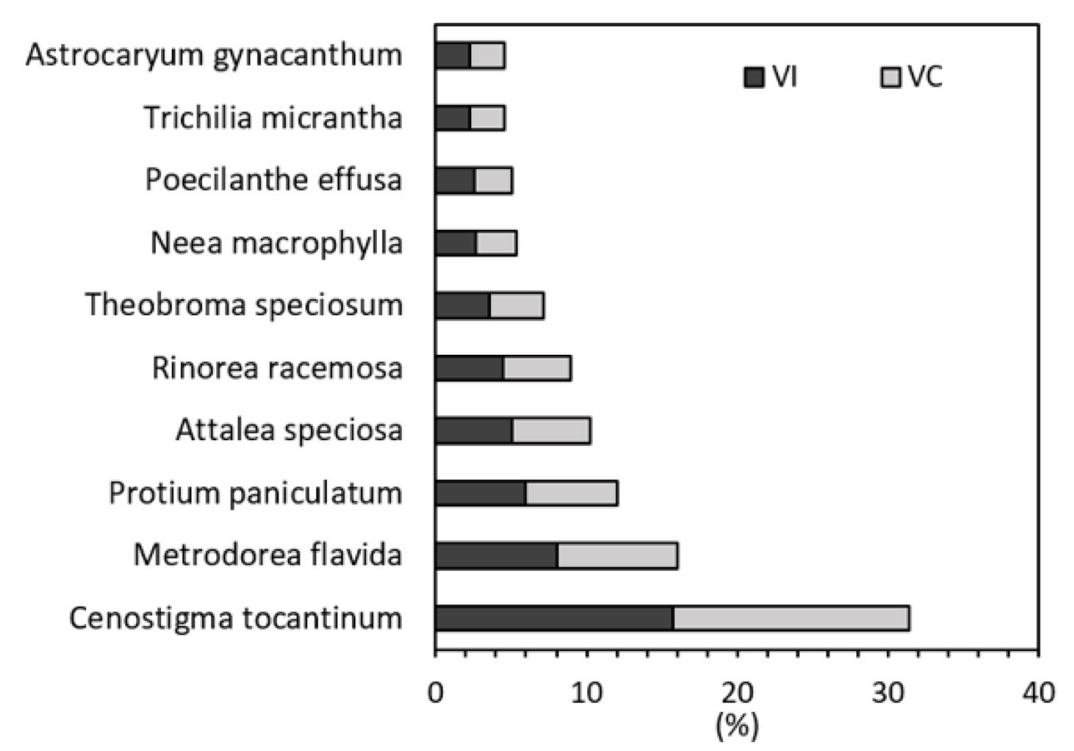

Figura 5 - Onze espécies mais importantes para área de estudo, rio Cinzento, Floresta Nacional do TapirapéAquiri, Pará, Brasil. VC= valor de cobertura (\%); VI= valor de importância (\%).

Ainda com relação a essa importância, de acordo com Pereira et al. (2011), espécies que apresentam alto valor de importância podem indicar funções-chaves que estão desempenhando nessa composição. Nesse sentido, os indivíduos supracitados e que apresentaram características interessantes, como o alto potencial ornamental (C. tocantinum, Attalea speciosa, e T. speciosum), artesanal (B. excelsa, A. speciosa, C. tocantinum, e C. catenaeformis) (Lorenzi, 1998; Carvalho, 2006; Guarim-Neto \& Silva, 2011), e alimentício (A. gynacanthum) desempenham um papel importante namanutenção ecológica, mas também apresentam boas características para o manejo.
É importante salientar que segundo os valores de VI e VC, o perfil florístico da área estudada é composto por três espécies que caracterizam esse ambiente, $C$. tocantinum, $M$. flavida, e P. paniculatum. A primeira é uma espécie amplamente distribuída em florestas de terra firme, sendo citada no próprio plano de manejo da unidade como um indivíduo mais bem distribuído na FLONATA (Brasil, 2006; Garcia et al., 2008). Enquanto a $P$. paniculatum e a $M$. flavida também são figuradas como espécies diversificadas $e$ comumente encontradas em regiões amazônicas (Vieira et al. 2015; Quaresma \& Jardim, 2011). 
Excetuando-se a C. tocantinum, dos 784 indivíduos, $16 \%$ correspondem a essa espécie, indicando que a representatividade da mesma tem sido favorecida pelas condições locais, fazendo com que ela domine o estrato ecológico existente para a área de estudo e desempenhe uma alta representatividade nessa comunidade. Uma das características desse indivíduo que propicia essa representação é o rápido crescimento e a facilidade de dispersão (Carvalho, 2006), ou seja, ela tende a ser menos susceptível à extinção local e há um possível esgotamento genético.

Essas características de rápido crescimento, facilidade de dispersão e a tendência ao menor esgotamento genético também são aspectos importantes para recuperação de áreas degradadas, o que tanto a $C$. tocantinum como a P.paniculatum apresentam, segundo Pott \& Pott (2002) e Warwick $\&$ Lewis (2009). Além disso, a C. tocantinum ainda é um indivíduo com potencial madeireiro utilizado em construções civis (Warwick \& Lewis, 2009), enquanto a $P$. paniculatum ainda tem em seu potencial características medicinais, como propriedades anti-inflamatórias e antibacterianas (De Almeida et al., 2015).

Nesse cenário, das onze espécies listadas como as mais representativas, cinco tiveram um potencial de uso que podem ser utilizados em composições com as mesmas características. $\mathrm{Na}$ área estudada, assim como em outras composições, a grande riqueza de indivíduos com potencial de uso eleva a capacidade do manejo. Desse modo, se for delimitado o uso das principais espécies em mais estudos, tem-se uma boa alternativa para aumentar o conhecimento, a fonte de renda de comunidades e sucessivamente a conservação $e$ valorização das espécies.

\section{Conclusão}

A comunidade de estudo apresentou uma estrutura característica de áreas de FOA, com uma grande concentração de palmeiras, árvores médias e pequenas quantidades de grandes árvores, indivíduos que demonstraram o alto grau de conservação da área. Em contrapartida, o valor de diversidade foi médio com uniformidade, indicando a existência de algumas espécies mais dominantes na composição estudada.
O perfil florístico da área indicou a relevância de onze espécies arbóreas, entre elas, cinco apresentaram um alto potencial, demonstrando que essa fitofisionomia possui características interessantes para novas perspectivas de manejo voltadas tanto para propriedades ornamentais, medicinais, de reflorestamento $e$ artesanais, assim como propriedades madeireiras.

As variáveis fitossociológicas consideradas neste estudo, como densidade, frequência $e$ importância das espécies, são relevantes para a criação de conhecimento na utilização do manejo racional. Contudo, são necessários mais estudos ecológicos e socioeconômicos que fortaleçam esse conhecimento para toda comunidade.

\section{Agradecimentos}

Ao Instituto Chico Mendes de Conservação da Biodiversidade (ICMBio) e à Fundação de Tecnologia Florestal e Geoprocessamento (FUNTEC-DF) pelo apoio a este projeto.

\section{Referências}

Brasil. 2006. Plano de Manejo Para Uso Múltiplo da Floresta Nacional do Tapirapé-Aquiri. 1 ed. IBAMA Instituto Brasileiro do Meio Ambiente e dos Recursos Naturais Renováveis. 453p.

Brandao PC, Souza AL, Quinet A \& de Mendonca BAF. Caracterização Estrutural e potencial florestal para o manejo comunitário da Floresta Nacional do Puras, Amazonia Ocidental/Structural characterization and forest potential for community management of Purus National Forest, Western Amazonia. Ciência Florestal, 30(4), 944-958, 2020.

Burgh Daly DC de \& Fine PVA, Martínez-Habibe MC. Burseraceae: a model for studying the Amazon flora. Rodriguésia, 63(1):21-30, 2012.

Carneiro MS, Assis WS, Souza UDV \& Dourado L. Deforestation governance in the Amazon from a Strategic Action Fields perspective. Ambiente \& Sociedade, 23(1), 2020.

Carvalho PER. 2006. Espécies Arbóreas Brasileiras. Embrapa Florestas: Colombo. 627p.

Cavalcanti JJV et al. 1999. Repetibilidade e número de avaliações necessárias à seleção de clones de cajueiroanão-precoce. Fortaleza: Embrapa-CNPAT, Boletim de Pesquisa. 12p. 
Colpini C, Travagin DP, Soares TS \& Silva VSM. Determinação do volume, do fator de forma e da porcentagem de casca de árvores individuais em uma Floresta Ombrófila Aberta na região noroeste. Acta Amaz, 39(1): 97-104, 2009.

CNCFlora. Centro Nacional de Conservação da Flora. Brasil <http://cncflora.jbrj.gov.br/portal>. Acesso em: 02/07/2020.

De Almeida $\mathrm{P}$ et al. Anti-inflammatory activity of triterpenes isolated from Protium paniculatum oilresins. Evidence-Based Complementary and Alternative Medicine, 2015.

Dionisio LFS. Efeitos a médio prazo da exploração seletiva no crescimento, mortalidade e recrutamento de Manilkara huberi (Ducke) A. Chev. em uma Floresta Amazônica. Sci. For., 48(1): 1-17, 2020.

Eiserhardt WL, Synning JC, Kissling WD \& Balsley $\mathrm{H}$. Geographical ecology of the palms (Arecaceae) determinantes of siversity and distribuitions across spatial scales. Ann Bot, 108(8):1391-1416, 2011.

Fernandes GGC, Ferreira LCO, Barros AI, Albuquerque AR \& Vieira AL. Estrutura populacional e distribuição espacial de Theobroma speciosum Wills. Ex Spreng (Cacauí) na Floresta Nacional do Tapirapé-Aquiri. Brazilian Journal of Development, 6(8): 56155-56163, 2020.

Ferreira TDS. Distribution and richness of rare tree species in Araucaria Forest fragments, along an altitudinal gradient, in Santa Catarina State, Brazil. Revista Árvore, 39(3): 447-455, 2015.

Finol H. La silvicultura en la Orinoquia Venezolana. Revista Forestal Venezolana, 14(25):37-114, 1975.

Gama JRV, Souza ALD, Calegario N \& Lana GC. Fitossociologia de duas fitocenoses de Floresta Ombrófila Aberta no município de Codó, estado do Maranhão. Rev. Árvore, 31(3): 465-477, 2007.

Garcia LC, De Moraes RP \& De Lima RMB. Determinação do grau crítico de umidade em sementes de Cenostigma tocantinum Ducke. Revista Brasileira De Sementes, 30(3): 172-176, 2008.

Guarim-Neto G \& Silva FHB. Plantas da Amônia MatoGrossense: O Cacauí - Theobroma speciosum Willd. ex Spreng (Malvaceae). Flovet, 3(1), 2011.

Gaston K.1994. Rarity. 1 ed. Chapman and Hall. 207p.

Hubbel SP. Tropical rain forest conservation and the twin challenges of diversity and rarity. Ecology and Evol, 3(10):3263-3274, 2013.

Hubbell SP \& Foster RB. 1986. Biology, change, history and the structure of tropical rain forest tree communities. p. 314- 329. In: J. Diamond \& T.J. Case (eds.). Community Ecology. Harper and Row: New York.
Joly CA et al. Florística e fitossociologia em parcelas permanentes da Mata Atlântica do sudeste do Brasil ao longo de um gradiente altitudinal. Biota Neotropica, 12(1): 125-145, 2012.

Lau AV, Ferreira GC \& Jardim MA. Fitossociologia e aspectos ecológicos da comunidade arbórea do Bosque Rodrigues Alves-Jardim Botânico Amazônia, Belém, Pará, Brasil. Revista Brasileira de Geografia Física, 13(2), 510-526, 2020.

Lorenzi H. 1998. Árvores brasileiras: manual de identificação e cultivo de plantas arbóreas nativas do Brasil. $2^{a}$ ed. Instituto Plantarum de Estudos da Flora Ltda. Nova Odessa, São Paulo, 368p.

Lima RC et al. Composition and structure of stretch of tropical forest in the Eastern Amazon. Ciência Rural, 5(4), 2021.

Lima SF, Matnii N \& Carvalho JOP de. 2000. Estrutura de uma floresta de terra firme na região de Marabá- PA: A posição do Mogno em relação ás outras espécies da comunidade. Comunicado técnico. $5 p$.

Magurran AE. 1988. Ecological diversity and its measurement. Princeton university press. 181p.

Malhi $Y$ et al. An international network to monitor the structure, composition and dynamics of Amazonian forests (RAINFOR). J. Veg. Sci., 13:439-450, 2002.

Miller PM \& Kauffman JB. Effects of Slash and burn agriculture on species abundance and composition of a tropical deciduous forest. Forest Ecology and Management, 103: 191-201, 1998.

Miranda IPA, Rabelo A, Bueno CR, Barbosa EM \& Ribeiro MNS. 2001. Frutos de Palmeiras da Amazônia. MCT INPA. 118p.

Moura V, Rosell EF \& Mascarenhas ARP. Análise fitossociológica de uma floresta ombrófila aberta em diferentse modelos de colonização da Amazônia. Nativa, 5(2):118-126, 2017.

Mueller-Dombois D \& Ellenberg H. 1974. Aims and methods of vegetation ecology. Jhon Wiley and Sons, Inc. $547 p$.

Nascimento AR, Felfili JM \& Meirelles EM. Florística e estrutura da comunidade arbórea de um remanescente de Floresta Estacional Decidual de encosta, Monte Alegre, Do, Brasil. Acta Bot. Bras, 18(3): 659-669, 2004.

Negrelle RRB. Attalea phalerata Mart. ex Spreng.: aspectos botânicos, ecológicos, etnobotânicos e agronômicos. Ciência Florestal, 25(4): 1061-1066, 2015.

Oliveira NA de \& Amaral IL do. Aspectos florísticos, fitossociológicos e ecológicos de um sub-bosque de terra firma na Amazônia Central, Amazonas, Brasil. Acta Amazonica, 35(1):1-16, 2005. 
Oliveira AND \& Amaral IL. D. Florística e fitossociologia de uma floresta de vertente na Amazônia Central, Amazonas, Brasil. Acta Amazonica, 34(1): 21-34, 2004.

Oliveira NA de, Amaral IL do, Ramos MBP, Nobre ad, Couto LB \& Sahdo RM. Composição e diversidade florístico-estrutural de um hectare de floresta densa de terra firme na Amazônia Central, Amazonas, Brasil. Acta amazonica, 38(4): 627-641, 2008.

Pereira LA, Sobrinho FAP \& Coata-Neto SVC. Florística e estrutura de uma mata de terra firme na reserva de desenvolvimento sustentável rio iratapuru, Amapá, Amazônia Oriental, Brasil. Floresta, 41(1):113-122, 2011.

Pielou EC. The measurement of diversity in different types of biological collection. J. Theoret. Biol., 13:131144, 1966.

Pott A \& Pott VJ. 2002.Plantas nativas para recuperação de áreas degradadas e reposição de vegetação em Mato Grosso do Sul. Embrapa Gado de Corte-Comunicado Técnico (INFOTECA-E).6p.

Quaresma AC \& Jardim MA. Formations of Coastal Forests in the Amazon and ecological relations with vascular epiphytes. Rev. árvore, 41(2): 410202, 2017.

Rodríguez-Estrella R \& Moreno MCB. Rare, Fragile Species, Small Populations, and the Dilemma of Collections. Biodiversity and Conservation, 15(1): 1621-1625, 2006.

Rocha AES \& Silva MFF . Aspectos fitossociológicos, florísticos e etnobotânicos das palmeiras (Arecaceae) de floresta secundária no município de Bragança, PA, Brasil. Acta Bot. Bras, 19(3):657-667, 2005.

Rolim SG, Couto HTZ, Jesus RM \& França JT. Modelos volumétricos para a Floresta Nacional do TapirapéAquirí, Serra dos Carajás (PA). Acta Amazonica, 36(1):107- 114, 2006.

Salles JC \& Schiavini I. 2 Estrutura e composição do estrato de regeneração em um fragmento florestal urbano: implicações para a dinâmica e a conservação da comunidade arbórea. Acta Botânica Brasilica 21: 223-233, 2007.
Salomão RP et al. As florestas de Belo Monte na grande curva do rio Xingu, Amazônia Oriental. Ciências Naturais, 2(3):57-153, 2007.

Schawartz G. Manejo sustentável de florestas secundárias: espécies potenciais no nordeste do Pará, Brasil. Amazônia: Ci. \& Desenv., 3(5):125-147, 2007.

Silva KE, Souza CR, Azevedo CP \& Rossi LMB. Dinâmica florestal, estoque de carbono e fitossociologia de uma floresta densa de terra-firma na Amazônia Central. Scientia Forestalis, 43(2): 193-201, 2015.

Silva JNM et al. 2005.Diretrizes para a instalação e medição de parcelas permanentes em florestas naturais da Amazônia Brasileira. 1 ed. EMBRAPA Amazônia Oriental. 68p.

Souza-Filho PWM et al. Four decades of land-cover, land-use and hydroclimatology changes in the Itacaiúnas River watershed, southeastern Amazon. Journal of Environmental Management, 167(1): 175-184, 2016.

Souza EM \& Sccoti MSV. Influência da Exploração Florestal nas Populações de Espécies Ameaçadas de Extinção na FLONA do Jamari, RO. Biodiversidade Brasileira, 10(3):64-73, 2020.

Vieira DS, Gama JRV, Oliveira MLR de \& Ribeiro RBS da. Análise estrutural e uso múltiplo de espécies arbóreas em florestas manejadas no médio vale do rio Curuá-Una, Pará. Floresta, 45(3): 465-476, 2015.

Warwick MC \& Lewis GP. A revision of Cenostigma (Leguminosae - Caesalpinioideae - Caesalpinieae), a genus endemic to Brazil. Kew Bulletin, 64(1): 135-146, 2009.

Xavier KRF, Andrade LA, Fabricante JR, Coelho MSE \& Assis FN. Impactos pós-fogo na regeneração natural em um fragmento de floresta ombrófila aberta no município de Paraíba. Revista Brasileira de Biociências Brasil, 9(3): 257-254, 2011.

\section{Biodiversidade Brasileira - BioBrasil. Edição Temática: PIBIC}

$$
\text { n. 1, } 2022
$$

http://www.icmbio.gov.br/revistaeletronica/index.php/BioBR

Biodiversidade Brasileira é uma publicação eletrônica científica do Instituto Chico Mendes de

Conservação da Biodiversidade (ICMBio) que tem como objetivo fomentar a discussão e a disseminação de experiências em conservação e manejo, com foco em unidades de conservação $e$ espécies ameaçadas.

ISSN: 2236-2886 